\title{
7. The theory of granularity
}

\section{FIRST, MARKOV'S BLANKET}

Andrei Andreyevich Markov was a Russian mathematician who died in 1922. Most of his work focused on the theory of stochastic process, which, in probability theory, is defined as a process involving the operation of chance. From this theory stems what Judea Pearl described in 1988 as the "Markov blanket," a subset of variables from which one may infer a random variable. Each Markov blanket is minimal, meaning that none of the variables can be removed without impacting the whole. It follows that each Markov set (e.g., a living organism) is made up of several elements, and that deleting one of them will change the set evolution.

Markov's sets explain why living things exist separately from others and develop in differentiated ways. ${ }^{1}$ Without blankets, biological elements would dissipate, creating one big mass of information in which there were no distinct entities. The blanket is what separates us from what isn't us.

But a Markov blanket is only observational; it does not explain why these sets of information remain unified. This is where Karl Friston, a British theoretical neuroscientist teaching at the University College of London, comes in. According to Friston, each organism is composed of several overlapping Markov sets. And here is the core of his theory: each of these sets resists a tendency to dissolve to align the actual world with its predictions. ${ }^{2}$ The best way for each set to achieve that result is to remain unified and act in such a way that the prediction comes true, which is minimizing "prediction error" or, as Friston says, minimizing free energy.

In this chapter, I will argue that blockchain's blanket is created by the blockchain nucleus's willingness to stand together to ensure survival. This chapter is inspired by Friston's free energy principle, which I realized only a few months

1 Judea Pearl, Probabilistic Reasoning in Intelligent Systems: Networks of Plausible Inference (Morgan Kaufmann Publishers, 1988).

2 Shaun Raviv, "The Genius Neuroscientist Who Might Hold the Key to True AI," Wired, November 13, 2018, https://perma.cc/9M44-45TG ("within us are blankets separating organs, which contain blankets separating cells, which contain blankets separating their organelles"). 
after I started working on it. Minimizing free energy explains why blockchain participants form a blanket by coming naturally together to ensure survival: it minimizes surprises. As Friston told me during our exchanges, "I concur with your sentiment that the nucleus would be subject to the same sorts of dynamics seen in other (non-vertical) forms of self-organisation," such as living organisms.

\section{BLOCKCHAIN'S LEGAL FICTION}

In this section, I introduce the theory of granularity and outline how it enables the application of antitrust law to blockchains. Transactional by nature, that theory aims to explain public permissionless blockchains beyond the simple cost reduction framework. It seeks to translate accurately the governing reality of such blockchains, creating for the purpose a new legal fiction that encapsulates blockchain without forcing it into inadequate boxes.

\subsection{Dynamics of Blockchain Governance}

The theory of granularity, to which one may want to provide a semantic explanation, frames blockchain governance as a new transactional institution. By doing so, it fills the gap created by the impossibility of applying the theory of the firm to public permissionless blockchains.

\subsubsection{Semantic explanation}

In "The Nature of the Firm", Ronald Coase distinguished between organizations and organisms. ${ }^{3}$ While firms are organizations, blockchains are clusters of organisms that, by nature, are spontaneous. Their functioning must be analyzed and understood this way so that antitrust and competition law can be properly applied when necessary.

The present chapter introduces the theory of granularity for the purpose. Generally, the notion of granularity defines the size of the smallest element in a system - that is, an organism. Thus, this theory aims to analyze the role played by each component of a blockchain. Unlike the firm, where vertical control is exercised over its components, blockchains are made up of horizontal governance mechanisms. This reinforces the importance of each organism,

3 See Ronald H. Coase, "The Nature of the Firm," Economica 4 (1937): 387. Friedrich Hayek, to whom Ronald Coase refers, called organisms "an order which is not made by anybody but which forms itself," see Friedrich Hayek, Kinds of Order in Society (Liberty Fund, 2013). 
as one cannot merely assume that they will follow one coordinated direction. ${ }^{4}$ One must then study blockchain's smallest organisms, the role they play and their dynamism. ${ }^{5}$ It is only by analyzing the granularity level that blockchain governance can be properly understood. ${ }^{6}$

\subsubsection{Understanding blockchain governance}

Blockchain is a space in which different forms of power are being exercised. However, unlike the firm, in which one exercises a power of command and control, I have explained that no single actor can entirely control a public permissionless blockchain. ${ }^{7}$ As a result, multiple interests can compete within the same blockchain; they may even be opposed. Blockchain "contribute[s] to the realization of a number of individual objectives which no one knows in their totality." "For that reason, one must study the different types of power that are generally found within public permissionless blockchains to understand which interests may eventually prevail over others. In doing so, we should keep in mind that "people who think the purpose of blockchains is to completely expunge soft mushy human intuitions and feelings in favor of completely algorithmic governance (emphasis on "completely') are absolutely crazy.",

I study blockchain power games by analyzing what I have described as the fifth blockchain level in Chapter 4: the governance layer. That level sits on top of more technical ones, and it appears to be central in defining the activities at the levels above. Furthermore, different constraints come into play in blockchain governance - namely, economic, political, logical, sociological, architectural and legal ones. Understanding how these constraints interact is a challenge; but it is essential in order to get a grip on who holds control over blockchain layer 1 and how that power is exercised over other participants. 2.1.2.

For an analysis of the role played by each blockchain participant, see section

5 David S. Evans, "Economic Aspects of Bitcoin and Other Decentralized Public-Ledger Currency Platforms," Coase-Sandor Institute for Law \& Economics Working Paper No. 685 (2014) (explaining that the analysis of public ledger platforms is "much more complicated" than typical open project projects).

6 When the granularity level is reached, it is impossible to break down the elements into smaller pieces.

7 See Christian Catalini and Joshua S. Gans, "Some Simple Economics of the Blockchain," MIT Sloan Research Paper No. 5191-16 (2019): 16 (arguing that, in the blockchain, each participant "can theoretically shape its evolution in a way that is proportional to its stake in the platform - e.g. in terms of computing power, storage, labor or capital dedicated to it").

8 Friedrich Hayek, Law, Legislation and Liberty (Routledge, 1973): 109.

9 Vitalik Buterin, "Notes on Blockchain Governance," Vitalik Buterin's website, December 17, 2017, https://perma.cc/34AB-ZL62. 
A distinction between all three categories of public permissionless blockchain participants is helpful in this regard - namely, between founders or core developers (I will often present them together for the sake of simplicity), users and miners. I show that although each blockchain has its specificities, the above-mentioned groups will use the same mechanisms to express their preferences, ${ }^{10}$ and will encounter the same limits if they act on their own. Eventually, their powers may suffer from four constraints that Lawrence Lessig described with his "pathetic dot theory": law, markets, social norms and architecture. ${ }^{11}$

As for private blockchains, I have explained that they mimic that structure to different degrees, depending on their original design. The closer they are to public permissionless blockchains, the less the theory of the firm will be transposable to them. The following developments then become relevant for public permissionless as well as private blockchains.

$10 \quad$ Jean Bacon et al., "Blockchain Demystified: A Technical and Legal Introduction to Distributed and Centralized Ledgers," Richmond Journal of Law \& Technology 25, no. 1 (2018).

11 See Lawrence Lessig, Code: And Other Laws of Cyberspace, Version 2.0 (Basic Books, 2006): 124 ("Norms constrain through the stigma that a community imposes; markets constrain through the price that they exact; architectures constrain through the physical burdens they impose, and law constrains through the punishment it threatens"). These constraints are "distinct, yet, they are plainly interdependent. Each can support or oppose the others," see id. at 123. Also, Primavera De Filippi, "Blockchain Technology and Decentralized Governance: The Pitfalls of a Trustless Dream," Decentralized Thriving: Governance and Community on the Web 3.0 (2019) (for an application of these four constraints to blockchain). 


\subsubsection{The power of founders and core developers ${ }^{12}$}

Blockchain founders and core developers are those who implement the original rules of a blockchain. ${ }^{13}$ They design the code software and determine which consensus protocol will be used. ${ }^{14}$

Although core developers work on the fourth level of blockchain - its infrastructure - they interact with other blockchain participants at the fifth level. Indeed, one may stress that the blockchain architecture limits their power, as they lose any form of direct control over other participants once they put the blockchain online. ${ }^{15}$ For most blockchains (but not all!) ${ }^{16}$ founders and core developers cannot unilaterally impose any changes ${ }^{17}$ or control who may

12 Founders and core developers are here put in the same category, although one may want to distinguish between them, as they are eventually in charge of the blockchain core code.

13 Vlad Zamfir, "Blockchain Governance 101," Goodaudience (blog), September 30, 2018, https://perma.cc/T4BY-NFE7. In short, core developers, also called "protocol developers," are to be differentiated from the developers building decentralized applications on top of the blockchain. Core developers are indeed "reviewing the code, proposing conceptual changes to the code, reviewing changes proposed by other coders, drafting new code and revising existing code, security-testing new code, compiling code into new releases, and communicating about the project with other developers, among others," according to Angela Walch, "In Code(rs) We Trust: Software Developers as Fiduciaries in Public Blockchains," in Techno-Social and Legal Challenges ed. Philipp Hacker, et al. (Oxford University Press, 2019): 9. See also Hanna Halaburda and Christoph Mueller-Bloch, "Will We Realize Blockchain's Promise of Decentralization?", Harvard Business Review, September 4, 2019, https:// perma.cc/PM6L-TT7H. Being in charge of the blockchain's original design gives them the opportunity, of course, to design it in a way to exercise more or less influence over the entire blockchain. For example, if core developers grant themselves the power to validate each transaction, they will exercise more control over the blockchain than under a Proof of Work mechanism.

14 See Michel Rauchs et al., Distributed Ledger Technology Systems: A Conceptual Framework (Cambridge Center for Alternative Finance, 2018): 55 (describing the different forms of protocol design and calling them anarchic, hierarchical, federal, plutocratic or democratic).

15 Primavera de Filippi and Greg Mcmullen, "Governance of Blockchain Systems: Governance of and by Distributed Infrastructures," Blockchain Research Institute and COALA (2018): 21; Raina S. Haque et al., "Blockchain Developers and Fiduciary Duty: An Ill-Fitting Framework," CLS Blue Sky (blog), June 21, 2019, https://perma .cc/WE5Q-QAGJ ("These developers are structurally unable to make decisions to impose changes on participants in a network").

16 See Ying-Ying Hsieh et al., "The Internal and External Governance of Blockchain-Based Organizations," in Bitcoin and Beyond: Blockchains and Global Governance, ed. Malcolm Campbell-Verduyn (2017): 8-9.

17 In fact, founders and core developers may simply submit changes to the majority of participants, see Raina S. Haque et al., "A Blockchain Development and Fiduciary Duty," Stanford Journal of Blockchain Law \& Policy 2, no. 2 (2019). One may suggest 
propose protocol updates. ${ }^{18}$ For instance, any Bitcoin Improvement Proposals must be voted upon, according to miners' computing power, before they get implemented. ${ }^{19}$ Indeed, "[ $\left.\mathrm{t}\right]$ he nature of Bitcoin is such that once version 0.1 was released, the core design was set in stone for the rest of its lifetime,"20 unless the majority agrees to change it.

that analyzing how core developers' proposals to update a blockchain protocol are being welcomed may prove useful in determining the degree of their power over other participants.

18 On some blockchains, the core developers are the only ones with the power to actually propose core code updates to the network. This is the case with Bitcoin Core, in which "maintainers" "have commit access and are responsible for merging patches from contributors. They perform a janitorial role merging patches that the team agrees should be merged. They also act as a final check to ensure that patches are safe and in line with the project goals. The maintainers' role is by agreement of project contributors," see "About Bitcoin Core," https://perma.cc/U696-6Y2J. It can also be that only the core developers can send emergency message to all the nodes on the network to ask them to adopt updates, as is the case on the Bitcoin blockchain, Angela Walch, "In Code(rs) We Trust: Software Developers as Fiduciaries in Public Blockchains," in Techno-Social and Legal Challenges eds. Philipp Hacker, et al. (Oxford University Press, 2019): 4, 13; see also Arthur Gervais et al., "Is Bitcoin a Decentralized Currency?," IEEE Security \& Privacy 12, no. 3 (2014), https://perma.cc/T6H7-AZ75. Nevertheless, Bitcoin core developers cannot impose these changes unilaterally, see Nick Tomaino, "The Governance of Blockchains," Medium, February 28, 2017, https://perma.cc/ MKW4-PHS7. The same is true for the Ethereum core developers, see Jean Bacon et al., "Blockchain Demystified: A Technical and Legal Introduction to Distributed and Centralized Ledgers," Richmond Journal of Law \& Technology 25, no. 1 (2018): 35. In short, protocol developers are not delegated with power or authority by other participants. There exists no agency relationship between developers and other participants in the network. Protocol developers lack the ability to bind other network participant, and no other network participant has the power to control the voluntary actions of protocol developers. They have no power to speak authoritatively for the community as a whole. On that, see Raina S. Haque et al., "A Blockchain Development and Fiduciary Duty," Stanford Journal of Blockchain Law \& Policy 2, no. 2 (2019): 139, 178. See Yessi Bello Perez, "The Controversies of Blockchain Governance and Rough Consensus," TNX, January 25, 2019, https://perma.cc/45W8-LXT7 (explaining that changes to the core code are submitted to nodes. They may vote to implement them, or to reject them). See also Sarah Azouvi et al., "Egalitarian Society or Benevolent Dictatorship: The State of Cryptocurrency Governance," 22nd International Conference on Financial Cryptography and Data Security (2018), https://perma.cc/S2GR-UJD5 (explaining that discussions regarding blockchain updates can nonetheless be centralized).

19 "Only developers can design and communicate proposed changes. As such, the developers speak and are listened to. Every BIP is authored by a developer," Jeffery Atik and George Gerro, "Hard Forks on the Bitcoin Blockchain: Reversible Exit, Continuing Voice," Stanford Journal of Blockchain Law \& Policy 1 (2018): 32.

20 Satoshi Nakamoto, "Re: Transactions and Scripts: DUP HASH160 ... EQUALVERIFY CHECKSIG," Bitcointalk.org, June 17, 2010, https://perma.cc/37PA -79WC (he insisted: "If Bitcoin catches on in a big way, these are things we'll want to 
The more participants are included in those voting procedures, the more decentralized that blockchain layer is..$^{21}$ The opposite is also true. For instance, Decred $^{22}$ and Tezos $^{23}$ are cryptocurrencies with more centralized governance systems. One of Tezos' principal characteristics is the ability to amend its consensus when necessary. ${ }^{24}$ The presence of off-chain and side-chain governance mechanisms, usually controlled by developers, should also be closely studied. $^{25}$

It remains that core developers do not control who can use the blockchain at the platform layer ${ }^{26}$ or who can build applications on top of it. ${ }^{27}$ That is because blockchain founders and core developers cannot impose changes on the blockchain code, interface, application, data or benefice. ${ }^{28}$ Their main role

explore in the future, but they all had to be designed at the beginning to make sure they would be possible later").

21 For an example of such a vote, see Yogita Khatri, "Zcash community votes to allocate $20 \%$ of network mining rewards to support development," The Block, January 29, 2020, https://perma.cc/ENA4-EG4M.

22 See Decred, https://perma.cc/VWE7-97DT.

23 L.M. Goodman, "Tezos — a self-amending crypto-ledger" (2014), https://perma .cc/978T-GZNJ.

24 According to which "protocol can be amended to reflect virtually any blockchain based algorithm."

25 See Vlad Zamfir, "Introducing Casper 'The Friendly Ghost'," Ethereum (blog), August 1, 2015, https://perma.cc/6A7Q-JMRX for a description of developments of "off-chain" and "sidechain" mechanisms. For a description of the need for these mechanisms, see Benito Arrunada and Luis Garicano, "Blockchain: The Birth of Decentralized Governance," Pompeu Fabra University, Economics and Business Working Paper 1608 (2018): 1.

26 Only permissioned blockchains permit that to be done, see Hanna Halaburda and Christoph Mueller-Bloch, "Will We Realize Blockchain's Promise of Decentralization?" Harvard Business Review, September 4, 2019, https://perma.cc/PM6L-TT7H. As for permissionless blockchain, as Tapscott and Tapscott put it, "nothing passes through a central third party; nothing is stored on a central server," Don Tapscott and Alex Tapscott, A Blockchain Revolution: How The Technology Behind Bitcoin Is Changing Money, Business, And The World (Penguin Books, 2016): 35.

27 Christian Catalini, "The Firm as a Nexus of Smart Contracts? How Blockchain and Cryptocurrencies Can Transform the Digital Economy," Yale Journal on Regulation: Notice \& Comment, June 7, 2017, https://perma.cc/KM4Y-963N (arguing that "the token can bootstrap the development of an entire innovation ecosystem where anyone can build novel applications on top of the underlying protocol without requiring permission from a network operator or intermediary").

28 Nick Tomaino, "The Slow Death of the Firm," The Control, October 21, 2017, https://perma.cc/H59E-WMZY. On Bitcoin, for instance, "developers who propose changes to the software can let the development team know. If it is a simple noncontroversial change then they will adopt it. If not, the developer is supposed to post the change and it will be adopted if there is a broad consensus in the community that it should be," see David S. Evans, "Economic Aspects of Bitcoin and Other Decentralized 
is thus close to that of "advisors," 29 but their influence is limited by blockchain participants' desire to maximize their own benefit, which may lead them, should they disagree with core developers, to refuse the implementation of new rules, to move to a rival ecosystem or to fork the blockchain. ${ }^{30}$ Social norms further limit them because they may fear not being influential enough to prevent hard forks.

Hard forks result in backward-incompatible software updates. When they do not obtain a sufficiently broad consensus among miners, ${ }^{31}$ hard forks cause the chain to split in two, permanently. Indeed, miners who do not follow the new block validation requirements will be unable to add their blocks to the latest version of the blockchain, as the core client will automatically reject them as non-compliant. Instead, a new chain of blocks will form, creating a split: two chains following different rules. These forks limit the core developers' willingness to act against the interests of other participants. ${ }^{32}$ And core developers may also fear soft forks, although to a lesser degree. Soft forks happen when

Public-Ledger Currency Platforms," Coase-Sandor Institute for Law \& Economics Working Paper No. 685 (2014): 1, 16. Also, Peder Østbye, "The Adequacy of Competition Policy for Cryptocurrency Markets" (2017), https://perma.cc/2DQ5-7PFU (stressing that the inability of blockchain's creators to alter the protocol or manipulate the transactions on the ledger is a key characteristic that should be taken into account in modeling competition law).

29 Don Tapscott and Alex Tapscott, Blockchain Revolution: How the Technology Behind Bitcoin Is Changing Money, Business, and the World (Penguin Books, 2016): 89; also, Raina S. Haque et al., "Blockchain Developers and Fiduciary Duty: An Ill-Fitting Framework," CLS Blue Sky (blog), June 21, 2019, https://perma.cc/WE5Q -QAGJ ("A protocol developers/s' ability to influence the welfare of the cryptocurrency holder - i.e., the value of the cryptocurrency - is therefore limited to proposing and advocating for a solution that the community may or may not adopt"). One may stress, nonetheless, that when these "advisors" are all working in the same space, they may collude to impose these changes more easily, see Vitalik Buterin, "The Meaning of Decentralization," Medium, February 6, 2017, https://perma.cc/6FP5-9L8Z.

30 Nick Tomaino, "The Slow Death of the Firm," The Control, October 21, 2017, https://perma.cc/H59E-WMZY. Generally, on the importance of product design as a way to control the use of the product, see Lawrence Lessig, Code: And Other Laws of Cyberspace, Version 2.0 (Basic Books, 2006). This explains why, after the DAO Ethereum fork in 2015, Ethereum and Ethereum Classic have survived: miners have switched from one to the other, depending on the gains they could realize. For more on the major Ethereum forks, see Ashley Viens, "Mapping the Most Important Ethereum Forks," Visual Capitalist, November 26, 2019, https://perma.cc/5BT7-ASWF.

31 See TechCrunch, "Blockchain Governance with Vlad Zamfir (Ethereum Foundation) at Ethereum Meetup 2018," published on August 21, 2018, YouTube, https://perma.cc/QZG6-R6XN (explaining that when a decision is considered to be illegitimate because it does not follow the norms, there is a high risk of forking).

32 See Dirk A. Zetzsche, Ross P. Buckley and Douglas W. Arner, "The Distributed Liability of Distributed Ledgers: Legal Risks of Blockchain," University of Illinois 
new rules are implemented, but when the blocks following the original rules are not rejected from the chain. These modifications are backward-compatible, accommodating miners who implement the change and those who do not.

Nevertheless, one should underline that these limits on core developers' power are linked to the decentralized nature of blockchain governance, which is not a necessary feature, but needs to be enacted. ${ }^{33}$ New blockchains may appear in which greater power is given to the founders and core developers. ${ }^{34}$ However, such blockchains will suffer from two inherent limits. First, the extent to which a (re)centralized blockchain could thrive remains to be seen. ${ }^{35}$ Such blockchains could deplete trust by confining power in the hands of a few, thus disincentivizing users from joining them. Second, a (re)centralized blockchain could function less efficiently than a truly decentralized one, because all its participants would no longer be in a position to improve it. This lack of efficiency, even if it only concerned certain types of transactions, could hinder these blockchains - which probably explains why, to this day, they have not prospered.

\subsubsection{The power of users ${ }^{36}$}

On permissionless public blockchains, users propose new transactions. Anyone can become a user. ${ }^{37}$ Users exercise substantial power over the blockchain, since their decision to use it (or not) is central to the blockchain's economic

Law Review (2018): 1361 (explaining that core developers may want to collaborate with other participants).

33 See Hanna Halaburda and Christoph Mueller-Bloch, "Will We Realize Blockchain's Promise of Decentralization?" Harvard Business Review, September 4, 2019, https://perma.cc/PM6L-TT7H.

${ }^{34}$ For instance, one could imagine a blockchain in which the consensus mechanism could involve the blockchain foundation in the validation of all transactions.

35 See David Evans, "Economic Aspects of Bitcoin and Other Decentralized Public-Ledger Currency Platforms" (2013): 16.

36 See Zetzsche et al., The Distributed Liability of Distributed Ledgers: Legal Risks of Blockchain (2017): 1384.

37 See Jean Bacon et al., "Blockchain Demystified: A Technical and Legal Introduction to Distributed and Centralized Ledgers," Richmond Journal of Law \& Technology 25, no. 1 (2018): 34. 
and social value. ${ }^{38}$ Their influence extends from influencing transaction fees ${ }^{39}$ to providing additional value by developing and using applications running on top of the platform layer. ${ }^{40}$ They can also force hard forks on the blockchain. ${ }^{41}$ However, their power is limited by the fact they cannot (easily) exercise coordinated control, as their actions are highly decentralized and spontaneous. ${ }^{42}$ This creates an architectural limit and makes their behavior primarily dependent on prices. ${ }^{43}$

38 See Thibault Schrepel, "Is Blockchain the Death of Antitrust Law? The Blockchain Antitrust Paradox," Georgetown Law Technology Review 3, no. 2 (2019): 296 (studying network effects on public and private blockchains). Also, Mappo, "Blockchain Governance 101", Medium, January 14, 2019, https://perma.cc/2LB8 $-32 \mathrm{NV}$ (explaining that users may indirectly hinder network effect by implementing unpopular decisions). Lastly, Vitalik Buterin, "The Limits to Blockchain Scalability," Vitalik Buterin's website May 23, 2021, https://perma.cc/6K2S-9455 ("The Ethereum blockchain has fully resolved consensus failures in ten hours; if your blockchain has only one client implementation, and you only need to deploy a code change to a few dozen nodes, coordinating a change to client code can be done much faster. The only reliable way to make this kind of coordinated social attack not effective is through passive defense from the one constituency that actually is decentralized: the users.")

39 Hanna Halaburda and Christoph Mueller-Bloch, "Will We Realize Blockchain's Promise of Decentralization?” Harvard Business Review, September 4, 2019, https:// perma.cc/PM6L-TT7H.

40 See Vitalik Buterin, "Notes on Blockchain Governance," Vitalik Buterin's website, December 17, 2017, https://perma.cc/ADP8-UCG4 (stressing that users' influence is limited by their potential lack of technical knowledge). Also, Balázs Bodó and Alexandra Giannopoulou, "The Logics of Technology Decentralization - The Case of Distributed Ledger Technologies," Amsterdam Law School Research Paper 2019-05 (2019): 1, 8 (developing the idea that core developers may use their technical skills to influence others). Lastly, see Vitalik Buterin, "The Most Important Scarce Resource is Legitimacy”, Vitalik Buterin's website, March 23, 2021, https://perma.cc/FZH7-ZZSV (explaining that despite the importance of core developers and validators, users have an important role to play in the ecosystem: providing projects with legitimacy).

41 Jake Frankenfield, "Hard Fork (Blockchain)," Investopedia, November 25, 2019, https://perma.cc/M9G2-7NVE.

${ }_{42}$ Vitalik Buterin, “The Meaning of Decentralization,” Medium, February 6, 2017, https://perma.cc/6FP5-9L8Z. In the words of Vitalik Buterin, blockchain communities are "using coordination problems" to their advantage, preventing one group of users from taking over, see Vitalik Buterin, "Engineering Security Through Coordination Problems," Vitalik Buterin's website, May 8, 2017, https://perma.cc/C3U5-KE9S.

43 See Lawrence Lessig, Code: And Other Laws of Cyberspace, Version 2.0 (Basic Books, 2006). 


\subsubsection{The power of miners ${ }^{44}$}

On permissionless public blockchains, miners validate transactions assembled into blocks. Any participant can become a miner. ${ }^{45}$ Miners follow the rules encoded in the fourth blockchain level (e.g., the Bitcoin Core client). ${ }^{46}$ They can comply with a different set of rules, but they will then waste computing power by producing an orphaned block, thus losing potential rewards. Following the main client's rules is miners' dominant strategy. ${ }^{47}$

If they coordinate their behavior, miners can influence a blockchain by realizing a 51 percent attack, ${ }^{48}$ thus forcing a soft fork. ${ }^{49}$ The risk is higher when miners are grouped into mining pools. ${ }^{50}$ In such a scenario, the blockchain pro-

44 See Evans, Economic Aspects of Bitcoin and Other Decentralized Public-Ledger Currency Platform, 1-2 (explaining that "the open-source public ledger platforms require another class of participants - the laborers who perform transaction processing - that require pecuniary compensation as an integral element of the protocol." These laborers are called "miners" within the blockchain sphere).

45 See Jean Bacon et al., "Blockchain Demystified: A Technical and Legal Introduction to Distributed and Centralized Ledgers," Richmond Journal of Law \& Technology 25, no. 1 (2018): 34.

46 Generally, it is said that "miners only have a limited role in the Bitcoin system, to secure the ordering of transactions, and they should NOT have the power to determine anything else, including block size limits and other block validity rules," on that see Vitalik Buterin, "Engineering Security Through Coordination Problems," Vitalik. ca, May 8, 2017, https://perma.cc/G2PC-W4LN.

47 It has been said, in fact, that "a miner's vote is better understood as its prediction of the collective stance of the broader miner community than as an expression of the voting miner's individual preference," see Jeffery Atik and George Gerro, "Hard Forks on the Bitcoin Blockchain: Reversible Exit, Continuing Voice," Stanford Journal of Blockchain Law \& Policy 1 (2018): 27-28.

48 See Primavera De Filippi and Benjamin Loveluck, "The Invisible Politics of Bitcoin: Governance Crisis of a Decentralised Infrastructure," Internet Policy Review 5, no. 3 (2016): 10-11.

49 Vitalik Buterin, "The Meaning of Decentralization," Medium, February 6, 2017 (arguing that the risk is particularly high when there is a strong community spirit); Vitalik Buterin, "Engineering Security Through Coordination Problems," Vitalik Buterin's website, May 8, 2017, https://perma.cc/C3U5-KE9S (underlining that "it is theoretically possible for miners to switch $99 \%$ of their hashpower to a chain with new rules," but in practice "quite hard to do"). More generally, see Taylor Pearson, "The Blockchain Man," Ribbonfarm, October 10, 2017, https://perma.cc/76FC-EK42 (contending that "appeals to authority, and perhaps violence, will be replaced by forking. If you disagree with a decision, you can fork a new blockchain").

50 Mining pools may indeed facilitate collusion, for instance, if all miners are in the same space. Nonetheless, whether there is a mining pool does not shift the paradigm as, in any case, such pools only reflect the will of their members. On the subject, see Craig S. Wright, "Proof of Work as it Relates to the Theory of the Firm," (2017), https://perma .cc/A984-BE62. Generally, individual miners do not have enough computing power to mine a block in widely used blockchains successfully - hence the existence of mining 
tocol is changed to loosen the rule-set enforced by full nodes. ${ }^{51}$ Such a change occurs when enough hashing power, or energy expended to mine a cryptocurrency, is devoted to it. ${ }^{52}$ The power of miners to start soft forks is nonetheless limited by both the blockchain's architecture ${ }^{53}$ and social norms - they must convince blockchain participants operating as nodes to run the new version of the software. ${ }^{54}$ Miners also suffer from market constraints, as initiating a soft fork may decrease the value of the tokens they own. ${ }^{55}$ The price mechanism also guides their actions, creating a strong market-related constraint. Finally, even if a fork were created, the new community would have the strenuous task of convincing other users to join it. ${ }^{56}$ For example, Bitcoin had been forked over 100 times at the time of writing. Over 30 of them are considered failures,

pools, see Philipp Hacker, "Corporate Governance for Complex Cryptocurrencies? A Framework for Stability and Decision Making in Blockchain-Based Organizations," in Regulating Blockchain. Techno-Social and Legal Challenges, ed. Philipp Hacker et al. (Oxford University Press, 2019): 32, https://perma.cc/DYZ2-THLJ. Miners may nonetheless, even at their individual level, prioritize transactions and exercise a form of power as such, see Nikhil Malik et al., "Why Bitcoin Will Fail to Scale?" (2019).

$51 \quad$ Full nodes are created where blockchain participants store an entire copy of the blockchain ledger on their computers, for more, see section 2.1.3. Underlining that "[t]here do exist non-mining full nodes," see Ethereum White Paper, 27.

52 Kristian Soltes, "The First Blockchain Antitrust Case. Or Is It?" Constantine Canon (Blog), May 29, 2019, https://perma.cc/RVB9-V96J.

53 See Ying-Ying Hsieh, Jean-Philippe Vergne and Sha Wang, "The Internal and External Governance of Blockchain-Based Organizations," in Bitcoin and Beyond Cryptocurrencies, Blockchains, and Global Governance (Routledge, 2019).

${ }_{54}$ See Jean Bacon et al., "Blockchain Demystified: A Technical and Legal Introduction to Distributed and Centralized Ledgers," Richmond Journal of Law \& Technology 25, no. 1 (2018): 37 (showing that there is no guarantee that nodes will agree to fork).

55 See Satoshi Nakamoto, "Bitcoin: A Peer-To-Peer Electronic Cash System," (2008), https://perma.cc/KJT5-KJ43. Also, Vlad Zamfir, "Blockchain Governance 101," Medium, September 30, 2018, https://perma.cc/D2VD-ZMMD (arguing that in the case of a fork, the trademark loses value).

56 John Light, "The Differences between a Hard Fork, a Soft Fork, and a Chain Split, and What They Mean for the Future of Bitcoin," Medium, September 25, 2017, https://perma.cc/ABQ3-GF7L. In the case of a soft fork, however, the blockchain remains compatible with previous versions despite the modification to its protocol, see Raina S. Haque et al., "A Blockchain Development and Fiduciary Duty," Stanford Journal of Blockchain Law \& Policy, 2 (2019): 162 (new transactions and new blocks can still be processed on the blockchain, as long as they do not violate the new rules of the protocol). Also, Christian Ewerhart, "Finite Blockchain Games," Economics Letters 197 (2020) (showing that "adherence to conservative mining or to the longest-chain rule constitute pure-strategy Nash equilibria", which explains why miners will generally be tempted to disregard forks). 
while another 29 projects are no longer capable of transacting. Among the remaining forks, just a few are considered valuable. ${ }^{57}$

\subsubsection{The blockchain power game}

This overall balance of power, common to all public permissionless blockchains, is the general analytical framework (as illustrated in Figure 7.1) within which to analyze whether one of these groups, on a case-by-case basis, has sufficient influence to qualify as control under antitrust or competition law.

\section{Participants}

\section{BLOCKCHAIN POWER GAME}
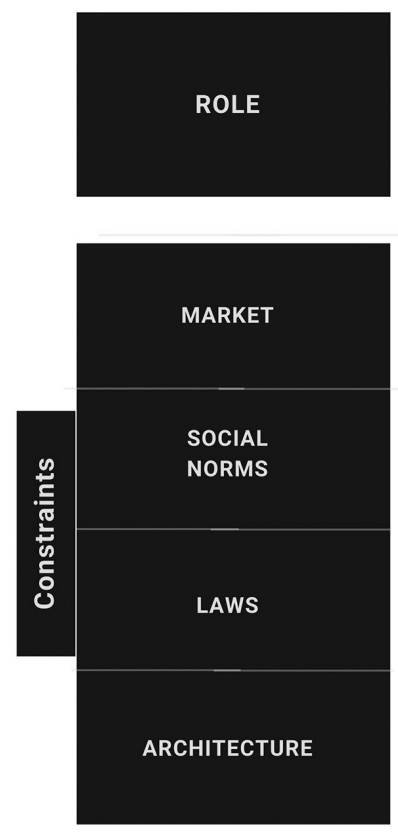
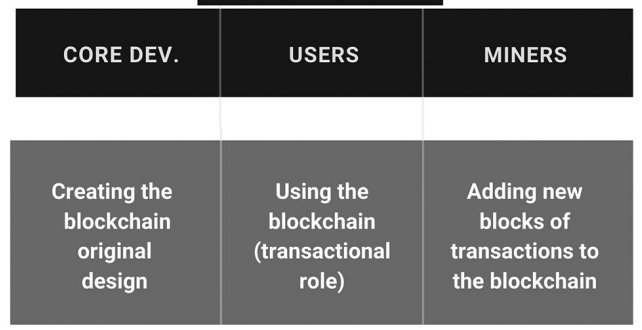

\begin{tabular}{|c|c|c|}
\hline $\begin{array}{c}\text { Fear of reducing } \\
\text { the blockchain } \\
\text { value by pushing } \\
\text { for bad changes }\end{array}$ & $\begin{array}{c}\text { Actions } \\
\text { (decentralized and } \\
\text { guided by the price } \\
\text { mechanism }\end{array}$ & $\begin{array}{c}\text { Fear of reducing } \\
\text { the blockchain } \\
\text { value (being } \\
\text { paid in tokens) }\end{array}$ \\
\hline $\begin{array}{c}\text { Fear the forks \& } \\
\text { fear of pushing for } \\
\text { changes against } \\
\text { the community's } \\
\text { general interest }\end{array}$ & $\begin{array}{c}\text { Fear of using a } \\
\text { blockchain with a } \\
\text { bad reputation }\end{array}$ & $\begin{array}{c}\text { Need the other } \\
\text { participants to } \\
\text { follow them in } \\
\text { case of a fork }\end{array}$ \\
$\begin{array}{c}\text { Laws may } \\
\text { eventually modify } \\
\text { incentives (tax, } \\
\text { copyright...) }\end{array}$ & $\begin{array}{c}\text { Leventually modify } \\
\text { incentives (tax, } \\
\text { copyright...) }\end{array}$ & $\begin{array}{c}\text { eventually modify } \\
\text { incentives (tax, } \\
\text { copyright...) }\end{array}$ \\
\hline $\begin{array}{c}\text { Cannot control the } \\
\text { use of the blockchain } \\
\text { \& cannot impose } \\
\text { changes to the } \\
\text { core code }\end{array}$ & $\begin{array}{c}\text { Constrained by the } \\
\text { blockchain original } \\
\text { design \& cannot } \\
\text { impose changes to } \\
\text { the core code }\end{array}$ & $\begin{array}{c}\text { Constrained by the } \\
\text { blockchain original } \\
\text { design \& cannot } \\
\text { impose changes to } \\
\text { the core code }\end{array}$ \\
\hline
\end{tabular}

Figure 7.1 A representation of blockchain power game

57 See Buy Bitcoin Worldwide, "Failed Forks," Buy Bitcoin Worldwide, https:// perma.cc/UX88-CDGN. 
On top of all that, core developers, users and miners may also store a copy of the blockchain ledger. When doing so, their computers are labeled as light nodes if they store only a subset of the blockchain ledger and full nodes if they store a copy of the entire blockchain. ${ }^{58}$

Although these nodes are passive and cannot be designated as actors in the blockchain, they ensure its integrity. This role carries power. First, blockchain participants who are nodes may alter their copy of the blockchain. ${ }^{59}$ Second, they may also (threaten to) validate blocks in which there is double spending. ${ }^{60}$ Their job is indeed to prevent users from spending the same token twice by allowing miners to verify the proposed transaction against a list of previous unspent transaction outputs. They protect blockchains value. However, their power is mainly limited by the fact that they cannot either control or influence transactions. ${ }^{61}$

This is the blockchain power game. It is well balanced, and technical solutions (called "layer 2" solutions) are constantly provided to maintain that balance. But these solutions are insufficient to maintain balance when different groups of blockchain participants come together to escape these constraints to the detriment of the broader ecosystem. When this occurs, they are exercising control over the blockchain.

\subsection{The Blockchain Nucleus}

Thus far, the theory of granularity has allowed me to determine the different forms of power enjoyed by blockchain participants. I must now detail how to

8 See Bacon et al., "Demystified", 19.

59 Philipp Hacker, "Corporate Governance for Complex Cryptocurrencies? A Framework for Stability and Decision Making in Blockchain-Based Organizations," in Regulating Blockchain. Techno-Social and Legal Challenges, ed. Philipp Hacker et al. (Oxford University Press, 2019) (explaining that blockchain participants operating as nodes are confirming authenticity through consensus). See Patrick Waelbroek, "An Economic Analysis of Blockchains," CESifo Working Paper Series 6893 (2018): 22 (blockchain participants operating as nodes have voting power on changes in the communication protocol.) Lastly, see Felix Irresberger, "Coin Concentration of Proof-of-Stake Blockchains," Leeds University Business School Working Paper 19-04 (2019): 1, 3 (explaining that nodes with more coins have more voting power).

${ }_{60}$ In such a scenario, blockchain participants operating as nodes would add these blocks to their local copy of the blockchain and broadcast them to other nodes on the network.

${ }^{61}$ See Mengerian, "The Market for Consensus," Medium, December 27, 2016, https://perma.cc/X4RV-5BNP ("Nodes enforce block validity rules to attempt to stay in consensus with the other nodes on the network. Nodes enforce block validity rules to try to influence properties of Bitcoin as a system"). 
identify a legal fiction controlling the blockchain. ${ }^{62}$ To this end, I explain what a blockchain nucleus is and then analyze its influence over other blockchain participants. I then describe how to define such a nucleus.

\subsubsection{Usefulness and challenges}

\subsubsection{The nucleus}

None of the three types of blockchain participants - core developers, users and miners - can impose their power on other groups to the point of taking complete control over the blockchain. Blockchains are indeed decentralized. They prevent the exercise of vertical power, and this differentiates them from firms in which a group, or sometimes even an individual, can control the other participants and "force them to collaborate," so to speak.

That being said, even with horizontal and decentralized governance, a group of participants may achieve a form of control over the blockchain by collaborating, by circumventing (some of) the constraints imposed on them, ${ }^{63}$ and by changing them in the long run. ${ }^{64}$

I contend that such a coalition exists for each blockchain (at least, for the surviving ones), ${ }^{65}$ and I call it the nucleus. The nucleus includes all the participants who have a personal interest (albeit transiently) to collaborate toward the same long-term goal: ensuring the blockchain's survival. ${ }^{66}$ Its members do not

62 The usefulness of antitrust and competition law is here presumed. Discussing antitrust legitimacy, see Oliver E. Williamson, "Allocative Efficiency and the Limits of Antitrust", The American Economic Review 59 (1969): 105, 116.

63 There are several solutions that allow the nucleus to strengthen its influence for example, quadratic voting, see Eric A. Posner and E. Glen Weyl, "Voting Squared: Quadratic Voting in Democratic Politics", Vanderbilt Law Review 68, no. 2 (2015): $441,441,446$ ("everyone votes on proposals (in the case of referenda) or candidates by buying as many votes pro or con as they want. The price they pay is the square of the number of votes they buy ... it give[s] proportional weight to people whose interests in a social outcome are stronger than those of other people").

64 Returning to Lawrence Lessig's pathetic dot theory, I am here studying the extent to which the dot may change the constraints. More specifically, I am analyzing how interactions between dots (blockchain participants) are contributing to modifying their environment.

65 See Vlad Zamfir, "Blockchain Governance 101," Goodaudience (blog), September 30, 2018, https://perma.cc/T4BY-NFE7 (for a blockchain to be successful, collaboration is needed).

${ }_{66}$ Within the firm, all participants have an interest in its survival. Within the blockchain, only those within the nucleus have a similar interest. The work of Karl Friston is helpful, by analogy, in understanding how organisms have an "interest" in staying together, see for instance Karl Friston et al., "Knowing One's Place: A Free-Energy Approach to Pattern Regulation", Journal of the Royal Society Interface 12 (2015): 1. A parallel may indeed be drawn between the principle of free energy, explaining how 
compete as they are, together, trying to maintain and expand their blockchain. Their short-term interests may diverge from time to time $e^{67}$ - for example, when two miners are racing to mine new blocks. ${ }^{68}$ Still, they seek to ensure blockchain integrity and systematically promote the same blockchain instead of other ones.

\subsubsection{Usefulness}

Assessing which participants have joined forces and are thus part of the nucleus is essential to determine who ultimately controls the blockchain. Put differently, it leads to identifying the participants that can be held liable for a breach of antitrust law when it is shown that they have anticompetitively exerted their influence. ${ }^{69}$ Identifying the nucleus amounts to creating a legal fiction to which the law can be applied, but also to which rights can be granted (see Figure 7.2).

The nucleus should indeed become a legal fiction that can be liable for anticompetitive practices, but also able to claim damages. In that regard, determining the nucleus size will prove central. It will prove useful in cases of anticompetitive practices directed at a blockchain nucleus. When a legal entity - whether a blockchain nucleus or a firm - infringes antitrust law and causes damages to another nucleus, the latter must have the means to introduce a legal action, stand by its rights and claim damages. Assigning liability and granting rights to blockchain ecosystems are thus two sides of the same coin.

biological systems maintain their order, and the blockchain nucleus, dealing with the question of how public permissionless blockchains maintain their existence. For further explanations on the idea of free energy and how cells stay together in the real space, see Shaun Raviv, "The Genius Neuroscientist Who Might Hold the Key to True AI," Wired, November 13, 2018, https://perma.cc/9M44-45TG.

67 Again, blockchain is not "a multiple team of horses drawing a vehicle under the control of a single driver," see Copperweld Corp. et al. v. Independence Tube Corp., 467 US 752 (U.S., 1984). Horses (participants) may go any direction they want, except if they have an interest not to - that is, if they are part of the nucleus.

${ }_{68}$ In fact, Satoshi Nakamoto suggested postponing competition for validating blocks under specific circumstances, see Satoshi Nakamoto, "Re: A Few Suggestions," Bitcointalk.org, December 12, 2009, https://perma.cc/JB2T-AQPT ("[w]e should have a gentleman's agreement to postpone the GPU arms race as long as we can for the good of the network").

${ }^{69}$ In a sense, identifying the blockchain nucleus relates to the theory of incentives as developed by Bengt Holmström and Paul Milgrom, "The Firm as an Incentive System," The American Economic Review 84, no. 4 (1994): 972. The nucleus allows for anticipation of the extent to which the participants cooperate with each other, or conversely, compete with each other. 


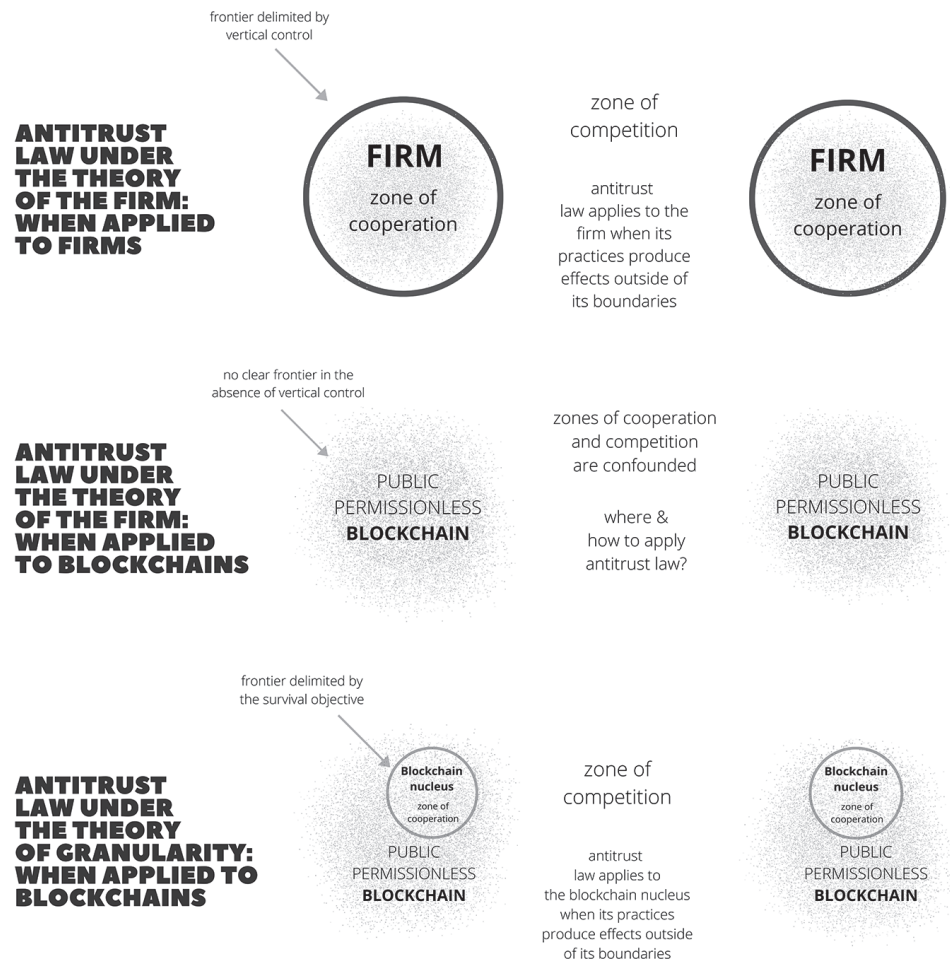

Figure 7.2 A representation of a blockchain nucleus

\section{DEFINING THE NUCLEUS SIZE}

Courts and antitrust agencies will face the task of determining the nucleus size. The further away a participant will be from the nucleus's center, the more difficult it will become to genuinely include her or him in the nucleus. With distance, it will prove harder to show that she or he could have influenced other participants' behavior. Only a case-by-case analysis can elucidate this question. This analysis should nevertheless be based on concrete and quantifiable frameworks to ensure legal certainty, limit legal errors and reduce regulatory costs. To this end, agencies should focus their investigation on economic agents' ability to exert a horizontal power of command and control. They 
should also consider their capacity to interfere with the blockchain's economic value and influence norms. ${ }^{70}$

Let me be more specific. The first element that should be factored in to determine which participants are part of the nucleus is the technical ability to exert a horizontal quasi-power of command and control. One must assess each blockchain's architectural characteristics to determine whether a few users may impose such decisions on others. The more a group of users can control others, the more they can single-handedly contribute to the blockchain's survival, and therefore be considered part of the nucleus. In fact, the original design of a blockchain can give one of the three groups of users more or less power. It can put them in charge of implementing the execution of transactions, designate them as miners or even enable them to change the design a blockchain's design unilaterally. Some blockchains might also use several mechanisms based on the platform layer to create governance (whether off-chain or side-chain). ${ }^{71}$

The second element is the ability of each participant to interfere with the blockchain's economic value. ${ }^{72}$ When some users govern the pricing structures, the blockchain's attractiveness or economic incentives, they have indirect control over the blockchain. This ability can be assessed by looking at technical elements. For instance, the capacity to change the size of each block, which may alter the number and types of transactions, is a sign of control. The same goes for the power to propose modifications to the core code to attract new participants. Finally, the more a participant has invested in the blockchain, the more he has an incentive to control its economic value. ${ }^{73}$ For that reason,

70 It may prove necessary to combine the analysis of these three different capacities to establish a body of corroborating evidence. Such methodology is often used in antitrust and competition law, see, for instance, Knauf Gips v Commission, C-407/08 P, European Court Reports: 2010 I-06375 (ECJ, 2010): para. 65; Monsanto Co. v. Spray-Rite Service Corp., 465 US 752, 768 (U.S., 1984) (stating that circumstantial evidence that reasonably tends to prove that the parties had a conscious commitment to a common scheme designed to achieve an unlawful objective could be used).

71 On the subject, see Vlad Zamfir, "Blockchain Governance 101," Goodaudience (blog), September 30, 2018, https://perma.cc/T4BY-NFE7 (arguing against the idea that blockchain nodes should automatically upgrade when an on-chain governance process decides on an upgrade because "blockchain governance is too important for us to let a small handful of cryptocurrency whales make arbitrary decisions").

72 Our analysis here borrows from the theory of incentive systems developed about the firm by Michael C. Jensen, "Theory of the Firm: Managerial Behavior, Agency Costs and Ownership Structure," Journal of Financial Economics 3, no. 4 (1976): 78. That being said, it differs from Jensen's theory because it does not focus solely on contract (the law), but also on blockchain digital architecture, norms and market incentives.

73 For that reason, core developers tend to be closer to the nucleus than simple users. These investments can be of a financial nature, but can also be measured in terms 
previous investments in a blockchain can show agencies where to look for the nucleus.

The third element is the ability to influence a blockchain's norms. ${ }^{74}$ Here, "norms" are defined as the "constraints imposed not through the organized or centralized actions of a state, but through the many slight and sometimes forceful sanctions that members of a community impose on each other" 75 - that is, the unwritten rules that one often feels compelled to follow. ${ }^{76}$ The more a participant can incentivize others to behave in a certain way - on pain of rejection from the community - the more they exercise control over the blockchain's general direction. ${ }^{77}$ For example, when core developers can influence other participants into accepting all of the modifications they would like to apply to the core (e.g., by arguing about the necessity for technical upgrades, security failures, bugs...), they effectively pilot part of the blockchain.

\section{THE THEORY OF GRANULARITY IN ACTION}

The theory of granularity would enable agencies to identify a blockchain's nucleus. It would thus permit the creation of a legal fiction to which antitrust can be applied. In turn, this would impose new obligations upon blockchain participants while simultaneously giving them new means to challenge anticompetitive behavior. This theory would make it possible to analyze relevant markets and market power in antitrust proceedings. The theory of granularity

of time and trust devoted to the blockchain. For instance, a participant with numerous smart contracts running on a blockchain has a strong interest in its survival. For an explanation of why having invested (in something) in the past creates a strong incentive to keep on investing, in particular because of sunk costs and path dependence, see Hai R. Arkes and Catherine Blumer, "The Psychology of Sunk Costs," Organizational Behavior and Human Decision Processes 35 (1984): 124 (explaining that "the sunk cost effect is manifested in a greater tendency to continue an endeavor once an investment in money, effort, or time has been made.")

74 See Vlad Zamfir, "Blockchain Governance 101," Goodaudience (blog), September 30, 2018, https://perma.cc/T4BY-NFE7 (discussing norms in blockchains).

75 Lawrence Lessig, Code: And Other Laws of Cyberspace, Version 2.0 (Basic Books, 2006): 340.

76 See Vlad Zamfir, "Blockchain Governance 101," Goodaudience (blog), September 30, 2018, https://perma.cc/T4BY-NFE7 (blockchain governance is not merely an issue of design; it is also a result of politics). Also, Ying-Ying Hsieh et al., "The Internal and External Governance of Blockchain-Based Organizations," in Bitcoin and Beyond: Blockchains and Global Governance, ed. Malcolm Campbell-Verduyn (Routledge, 2017) (norms may also come from outside the blockchain - for instance, from media or general public interest).

77 See Nigel Dodd, "The Social Life of Bitcoin," Sage Journal 10 (2017) (studying the influence of norms in the Bitcoin blockchain). 
would also make it possible to impute anticompetitive practices to a given set of blockchain participants.

\subsection{Market Analysis}

Public permissionless blockchains are horizontal and decentralized. The absence of borders makes it very difficult to apply traditional competition policy tools to them - including market definition. In turn, this makes it almost impossible to determine whether a given participant can exert any market power. The theory of granularity could help antitrust agencies and courts to overcome both difficulties.

\subsubsection{Relevant markets}

Two-sided markets have been the object of numerous studies, ${ }^{78}$ including a great deal about market definition. ${ }^{79}$ At this point in time, and following the Supreme Court's American Express decision, courts and agencies often consider that there is a single market encompassing both sides of two-sided transaction markets - that is, those in which the two sides transact directly with one another through an intermediary. ${ }^{80}$ In that case, the Supreme Court considered that both sides - merchants and cardholders - were part of the market for

78 The first article on the subject was Jean-Charles Rochet and Jean Tirole, "Platform Competition in Two-Sided Markets," Journal of the European Economic Association 1, (2003): 990. At the time of writing, it has been cited 4483 times according to Google Scholar, including 2400 times since January 1, 2015. See also Melvyn Weeks, "The Evolution and Design of Digital Economies" (2018): 27 (explaining that "economists and computer scientists are learning how to adapt the tools developed to build and understand the dynamics of centralised marketplaces" to decentralized markets).

79 See Lapo Filistrucchi et al., "Market Definition in Two-Sided Markets: Theory and Practice," Journal of Competition Law and Economics 10, no. 2, (2014): 293 (introducing a conceptual framework for defining relevant markets in two-sided markets). More generally, see Louis Kaplow, "Why (Ever) Define Markets?" Harvard Law Review 124, (2010): 81. For an answer, see Gregory J. Werden, "Why (Ever) Define Markets: An Answer to Professor Kaplow," Antitrust Law Journal 78 (2012): 729.

80 According to Ohio v. American Express Co., 585 U.S. 138 S. Ct. 2274, 201 L. Ed. 2d 678 (2018) ("The key feature of transaction platforms is that they cannot make a sale to one side of the platform without simultaneously making a sale to the other"). The Supreme Court held that they should be called "supplying only one product transactions" (internal citation omitted) for that reason, stressing that "[i]n two-sided transaction markets, only one market should be defined." 
credit card transactions. ${ }^{81}$ The European Commission, in its Android decision, confirmed the same principle. ${ }^{82}$

This has important implications when it comes to studying market power. Indeed, once they have identified the relevant product (or service) market, antitrust agencies usually study market power by identifying substitutes. ${ }^{83}$ Such an analysis is made possible because centralized two-sided platforms or aggregators are often centered around one or a few products or services. For instance, Uber offers one easily identifiable service. Other transporters, such as Lyft, are part of the same relevant market.

The way agencies define the market is also crucial when it comes to analyzing potential anticompetitive effects. When they consider that two sides of the platform or aggregator are part of the same relevant market, anticompetitive effects on one side can be compensated by procompetitive effects on the other. Such logic cannot be applied when two sides of a platform or aggregator are considered two relevant markets.

With that logic in mind, one can see how defining relevant markets is more complex when it comes to public permissionless blockchains. As I explained in Chapter 3, one may distinguish between three major types of blockchain applications. ${ }^{84}$ The first allows only one type of application - namely, cryptocurrencies. They compete with each other. Blockchain 2.0 permits the execution of smart contracts which can automate an almost infinite number of transactions. For that reason, all blockchain 2.0 platforms do not compete together, as they may automate transactions of a potentially very different nature. They cannot be easily shoehorned into a single product market. Finally, blockchain 3.0 makes it possible to do everything a computer can do, but in a decentralized and distributed fashion. ${ }^{85}$ The creators of such blockchains have no control over which products and services will ultimately be offered. Thus, one cannot consider such blockchains as part of a single relevant market. For example, unlike smartphone or computer operating systems, blockchains 3.0 are not intended to work on pre-defined types of products (e.g., smart-

${ }^{81} I d$., "[t]ellingly, credit cards determine their market share by measuring the volume of transactions they have sold."

82 "Google Android case" in Google LLC. v. Commission, Case AT.40099, C/2018/4761 (EC, 2018): para. 213, "for the definition of the relevant market, demand-side substitution constitutes the most immediate and effective disciplinary force on the suppliers of a given product."

${ }^{83}$ Id., see also Jon Baker, The Antitrust Paradigm: Restoring a Competitive Economy (Harvard University Press, 2019): 185.

84 Melanie Swan, Blockchain: Blueprint for A New Economy (O'Reilly Media, 2015): Preface, IX.

85 Id. 
phones). They can run wherever the Internet is available and they may even allow the subsequent development of operating systems.

As a result, public permissionless blockchains pose new challenges in terms of market definition that cannot be addressed by transposing the analyses of centralized two-sided platforms, as they are transactional infrastructures on top of which any types of products or services can be built freely. The theory of granularity, on the contrary, makes it possible to identify a blockchain nucleus and to provide both a theoretical and practical answer to these market definition issues.

Once the blockchain nucleus has been identified, one may define the types of activities around which it is built - that is, all activities ensuring the blockchain's survival. This makes it possible to identify substitutable blockchains (and centralized products or services) on the demand side. Blockchains may operate in several relevant markets, just as centralized platforms do. Focusing on the blockchain nucleus then allows us to delimit the geographical market (the area in which entities compete) by identifying all the substitutable products and services from the user's point of view.

\subsubsection{Market power}

For many years, academic articles have insisted on a disconnect between static legal analysis and the dynamic nature of digital markets. ${ }^{86}$ Public permissionless blockchains exacerbate this problem because their borders are not as stable as those of firms. Public permissionless blockchains have no entry or exit door, freeing their participants to move from one blockchain to another. This flexibility should lead agencies to rethink how they analyze market power. ${ }^{87}$

The theory of granularity helps overcome this difficulty by grouping participants within a single legal fiction. Comparing the different nuclei in the same relevant market leads to establishing their market power. And of course, one will be required to keep in mind that entry barriers may be much lower in blockchain ecosystems than in more traditional markets. To be sure, some

86 For instance, see J. Gregory Sidak and David J. Teece, "Dynamic Competition in Antitrust Law," Journal of Competition Law \& Economics 5, (2009): 581, 582 (underlining that antitrust and competition law has been static for several decades already); also Jan Krämer and Michael Wohlfarth, "Market Power, Regulatory Convergence, and the Role of Data in Digital Markets," Telecommunications Policy 42, no. 2 (2017): 154, 168 (underlining that, for instance, the dynamic nature of digital markets is per $s e$ in conflict with static approaches to define the relevant market); Michael O. Wise, "Antitrust's Newest New Learning Returns the Law to its Roots: Chaos and Adaptation as New Metaphors for Competition Policy," Antitrust Bulletin 40, no. 4 (1995).

87 Generally speaking, as has been stressed by Lawrence Lessig, "an unmovable, and unmoving, target of regulation, then, is a good start toward regulability," Lawrence Lessig, Code: And Other Laws of Cyberspace, Version 2.0 (Basic Books, 2006): 139. 
will remain, mostly for technical reasons (one will be required to learn about blockchain functioning and characteristics). Still, most of today's entry barriers will be removed, since access to dominant blockchains with significant market power will be open.

\subsection{Anticompetitive Practices}

The theory of granularity enables agencies to analyze the effects of practices by recreating an "inside" and "outside" (the nucleus), which is essential for antitrust analysis. Ultimately, it enables them to assign liability to the nucleus while also granting rights to its participants.

\subsubsection{The assessment of practices}

Firms decide internally to partake in collusive agreements or monopolization practices, but antitrust law is mostly concerned with such behavior's external effects. ${ }^{88}$ That distinction between the effects inside and outside the firm guides the analysis of all potential practices. This prevents the application of antitrust law to public permissionless blockchains. The absence of clearly defined boundaries for such blockchains at the platform layer precludes a distinction between what is inside or outside. Analyzing practices within blockchain ecosystems becomes immensely complex for that reason.

The delimitation of a blockchain nucleus reintroduces the possibility of analyzing internal and external effects, since it recreates borders - namely, inside and outside the legal fiction. One can easily understand this through the example of collusion. As entities cannot collude with themselves, one needs to delimit their boundaries to analyze whether a collusive practice has occurred. In that regard, only agreements between two different nuclei should be worrisome. Agreements between blockchain participants outside of any nucleus, de facto lacking any ability to control, should not trigger antitrust concerns.

The same goes for monopolization and abuse of dominance cases. The theory of granularity makes it possible to define a legal fiction whose market power will be assessed in relation to others. In turn, this enables agencies to determine whether a legal fiction abused its power by analyzing the external effects of its behavior. This analysis is not possible when blockchains are seen only from a distance, because blurry decentralized entities in which no one exercises a power of command and control have no visible frontiers. The theory of granularity fixes that.

88 Thibault Schrepel, "Antitrust Conversations with Nobel Laureates," Concurrentialiste Review (2018): 14. 


\subsubsection{The assignment of liability}

A firm controls its behavior on the market. That is true both for firms in their classic form and for firms that operate digital products and services. As Jean-Charles Rochet and Jean Tirole stressed in 2003, "platforms are controlled by competing entities, either profit-maximizing firms or not-for-profit associations. ${ }^{\circ 99}$ This is because platforms such as Microsoft are matchmakers: they allow app developers to find consumers and vice versa. The same is true for aggregators such as Facebook, on top of which there is no app developing, but whose service is to collect a critical mass of users on one side to leverage access on the other. ${ }^{90}$ They keep control of their practices, regardless of their business model.

Decentralized platforms are different because a few users cannot unilaterally decide on a strategy. For that reason, blockchains differ significantly from the model introduced by Rochet and Tirole, as practices emerge on them rather than being imposed. ${ }^{91}$ The decentralized and horizontal nature of blockchain raises two important antitrust-related issues: characterizing anticompetitive practices and identifying the person(s) liable for them. The theory of granularity introduced in this chapter provides an answer to both.

I have shown that the behavior of participants in public permissionless blockchains may be influenced in three ways by the nucleus: (1) the technical ability to enjoy a restricted and horizontal quasi-power of command and control; (2) the ability to interfere with the blockchain's economic value; and (3) the ability to influence the blockchain's norms. As a result, the nucleus

89 Jean-Charles Rochet and Jean Tirole, "Platform Competition in Two-Sided Markets," Journal of the European Economic Association 1 (2003): 1000.

90 For further thoughts on the distinction, see "Platforms Versus Aggregators, What About Amazon? Walmart Buys Flipkart", Stratechery, May 10, 2018, https://perma.cc/ E9T5-RB9E. Studying the implications of the distinction in antitrust law, see Thibault Schrepel, "Platforms or Aggregators: Implications for Digital Antitrust Law," Journal of European Competition Law \& Practice 12 (2021): 1.

91 Blockchains are no "islands of conscious power," D.H. Robertson, Control of Industry: Cambridge Economic Handbooks IV (Nisbet, 1923): 385, coining the terms to describe firms. No overall conscious strategy can be imposed on blockchain. It is problematic to the extent that objective intent is usually inferred from the implementation of practices, which no longer seems possible here. Generally, on the role of intent in antitrust and competition law, see Marina Lao, "Aspen Skiing and Trinko: Antitrust Intent and Sacrifice”, Antitrust Law Journal 73 (2005): 171, 208 (discussing the role of intent in the Aspen Skiing and Trinko cases); Nicolo Zingales, "Antitrust Intent in An Age of Algorithmic Nudging," Journal of Antitrust Enforcement 7 (2019): 386, 387 (proving the existence of a subjective intent is not required in antitrust and competition law, but proving objective intent is). 
participants are to be held liable for all illegal conduct committed within the nucleus or the perimeter that it can control or influence to a great degree. ${ }^{2}$

Agencies and courts will have to decide on the width of that perimeter on a case-by-case basis. ${ }^{93}$ By analogy with the American and European liability regimes for parent companies, ${ }^{94}$ agencies will have to prove each time that the nucleus had the power to influence other participants, and that it actually exercised that influence. ${ }^{95}$ However, the nucleus participants should not be held liable for practices committed outside of the perimeter they can influence. ${ }^{96}$ This absence of liability is not problematic, because blockchain participants outside that perimeter are submitted to different constraints that deprive them of any power to coordinate their actions effectively to change the blockchain direction or abuse their power with that aim. The lack of control should logically lead to a lack of liability. For example, one occasional miner will not be included in a blockchain nucleus, but this should not be troubling as that miner cannot influence the blockchain (and infer or stop anticompetitive behaviors).

92 One must distinguish between different degrees of distance with the nucleus that is, different degrees of collaboration or competition with it. This is equivalent to analyzing one participant's ability to compete with other participants by moving away from the nucleus when desired. This capacity is a key criterion in antitrust and competition law for determining the extent to which economic agents are undertaking, see Coöperatieve Vereniging Suiker Unie v. Commission, Joined Cases 40-48, 50, 54-56, 111, 113 and 114-73, European Court Reports: 19751663 (ECJ, 1975): para. 173. The CJEU has frequently repeated this rule, see, for instance, T-Mobile Netherlands $B V$, KPN Mobile NV, Orange Nederland NV and Vodafone Libertel NV v. Raad van bestuur van de Nederlandse Mededingingsautoriteit, Case C-8/08, European Court Reports: ECLI:EU:C:2009:343 (ECJ, 2009): para. 32, Allgemeine Elektrizitäts-Gesellschaft AEG-Telefunken AG v. Commission, Case 107/82, European Court Reports: 19833151 (ECJ, 1983): para. 49. As such, the further a participant is from the nucleus, the less that nucleus will be able to control it.

93 Some participants may indeed collaborate to ensure the blockchain survival in a majority of cases - they constitute the nucleus's nearby perimeter - while other participants may compete regularly with the nucleus, especially if they have a personal interest in the survival of another blockchain.

94 See Carsten Koenig, "Comparing Parent Company Liability in EU and US Antitrust and Competition Law," World Competition 41, no. 01 (2018) (explaining that in the United States, courts rely less on assumptions to infer liability to parent companies than they do in Europe).

${ }_{95}$ Alliance One International, Inc., formerly Standard Commercial Corp. and Others v. European Commission, Case T-24/05, European Court Reports: 2010 II-05329 (ECJ, 2010).

96 Vitalik Buterin, “Control as Liability”, Vitalik Buterin’s website, May 9, 2019, https://perma.cc/SR7C-9M43 ("every bit of control you have is a liability"). Liability in blockchain must stop where control stops. 
Finally, for fine calculation, the nucleus will be subject to the same rules applied to firms. The turnover realized by the nucleus, based on all its transactions, will serve as a basis for calculating sanctions. ${ }^{97}$

\section{A WIN-WIN THEORY}

The creation of a legal fiction around blockchain nuclei will benefit both antitrust and blockchain communities. By facilitating the enforcement of the rule of law, blockchain participants will indeed be able to enforce antitrust laws or be sanctioned when infringing them.

\subsection{A Win for Antitrust}

The theory of granularity helps create a legal fiction for public permissionless blockchains and private ones (whose governance is not vertical). Surely, other legal fictions will be proposed in the coming years. Regardless of its name, creating a legal fiction is a prerequisite for applying the rule of law to blockchain layer 1 . The ability to do so is crucial.

First, the creation of a legal fiction ensures that blockchains do not escape antitrust enforcement for theoretical reasons. This is a prerequisite before discussing the technical barriers to enforce antitrust against illegal practices (see the following chapters). Second, assigning liability to the right entity ensures that whoever controls blockchains will have a strong(er) incentive to comply with legal requirements. The urge to play by the rules is always stronger when one knows that the rules could actually be enforced. As such, antitrust will not only protect actors that lie outside of blockchain ecosystems; it will also protect those inside the blockchain who cannot stop the anticompetitive practices. Antitrust will free blockchain layer 1 from these practices.

\subsection{A Win for Blockchain}

Creating a distinct legal fiction centered on blockchains' nucleus will present an important step forward for related ecosystems.

First, the creation of such fiction will attribute rights to blockchains' nuclei. This will legitimize collaboration between blockchain participants in the nucleus that would otherwise have been prohibited. Indeed, I have explained

97 See, for example, MK Manoylov, "Bitcoin Transaction Values and Fees Grew in 2020's Third Quarter," The Block, October 10, 2020, https://perma.cc/3GQK-8EWL (during the second quarter of 2020, "users transacted more than $\$ 225 \mathrm{~B}$, posting an average of $\$ 2.4 \mathrm{~B}$ a day"). Even a small percentage of this value represents a very significant and dissuasive sum of money. 
that antitrust law defines a legal fiction (e.g., the firm) and then applies only to the effects that occur outside of it. Decisions that produce an effect outside of the blockchain nucleus will be submitted to antitrust law. In contrast, decisions taken by the nucleus whose effects are purely internal to that entity will be exempt from antitrust scrutiny. ${ }^{98}$

Second, creating a legal fiction will increase legal certainty pertaining to the application of antitrust law and regulation. Decades of research suggest that doing so will encourage investments, ${ }^{99}$ and will make entrepreneurs want to "embark" on the creation of innovative products and services. ${ }^{100}$ Blockchain communities say so themselves: regulatory issues and accompanying legal uncertainty are the most important reasons preventing greater investment and adoption of blockchain technology. ${ }^{101}$ The sooner a legal fiction is created, the better for the ecosystem. In its absence, one could imagine court decisions holding all blockchain participants liable for wrongdoings, even though most of them will not have the power to prevent these illegal practices.

Finally, the creation of a legal fiction will give the nucleus the right to institute legal actions and claim damages in cases of antitrust violation, whether caused by another nucleus or a non-blockchain entity. Going back to Christopher Stone's writing, blockchain's legal fictions will be able to institute legal actions in their name; courts will calculate injury to them, and relief will be run to their benefit. For example, one could imagine that a blockchain layer 1 (illegally) excluded from the market by another blockchain that engaged in predatory pricing could introduce a valid claim before the courts or antitrust agencies. In the following chapters, I will explain how this will play out when it comes to collusion and monopolization practices.

98 See Carla L. Reyes, "If Rockefeller Were a Coder," George Washington Law Review 87, no. 373 (2019): 428.

99 Avinash Dixit, "Entry and Exit Decisions Under Uncertainty," Journal of Political Economy 97, no. 3 (1989): 620; Robert S. Pindyck, "Irreversibility, Uncertainty, and Investment," Journal of Economic Literature 29 (1991): 1110; Avinash Dixit and Robert S. Pindyck, Investment Under Uncertainty (Princeton University Press 1994); Ricardo J. Caballero and Robert S. Pindyck, "Uncertainty, Investment, and Industry Evolution," International Economic Review 37, no. 3 (1996): 641; Nicholas Bloom et al., "Uncertainty and Investment Dynamics," The Review of Economic Studies 74, no. 2 (2007): 391.

100 Edmund S. Phelps, Mass Flourishing: How Grassroots Innovation Created Jobs, Challenge, and Change (Princeton University Press, 2013): 206.

101 See PwC, "PwC's Global Blockchain Survey 2018: Blockchain is Here. What's your Next Move?" (2018): 4, 11, https://perma.cc/PK33-RCD3 (48 per cent of survey respondents indicated that the lack of regulatory certainty is preventing the adoption of blockchain. This was ranked as the first reason). 
For all these reasons, creating an antitrust-related legal fiction will be invaluable for blockchain ecosystems and, ultimately, for decentralization. It will protect them from illegal practices that could hinder blockchain's capacity to decentralize the economy. There is no doubt that centralized companies will multiply illegal behaviors toward blockchain ecosystems in the years to come, as we will see in the coming chapters. Being recognized as a legal entity will allow them to protect their interests and innovate toward decentralization.

\section{CHAPTER SUMMARY AND BEYOND}

In this chapter, I have used the theory of granularity to open the blockchain "black box." First, I have discussed blockchain governance and shown how the influence of different participants neutralize their position. As no blockchain participant can control the blockchain by itself - and ensure its survival - I have explained that a group of participants may want to come together to achieve common goals. By doing so, they free themselves from other participants' constraints and end up forming the blockchain nucleus.

The blockchain nucleus gives rise to an entity that should benefit from rights, but could also be held liable for illegal conducts. I have shown how this would work by analyzing relevant markets and market power, evaluating anticompetitive practices and assigning liability.

Now that I have discussed antitrust law's applicability, I move on to exploring the anticompetitive practices that could be implemented thanks to blockchains. That is, I explore the application of antitrust law to different forms of behavior that could take place within blockchain ecosystems. I start with collusive agreements and show that blockchain may actually help agents to implement (more) effective cartels. 